the literature. In addition, no previous reports of markedly increased serum AFP levels, a finding usually associated with hepatoblastomas, have been described in patients with $\mathrm{MH}$, and only mildly increased serum AFP levels have been reported (the highest being $7500 \mathrm{ug} / \mathrm{L}$ ). In conclusion, MH may present with markedly increased levels of serum AFP, mimicking hepatoblastomas, and may also be part of the spectrum of findings in patients with BWS.

\section{Placental Pathology}

741 QUALITATIVE AND QUANTITATIVE IMMUNOHISTOCHEMICAL EVALUATION OF INFLAMMATORY CELL INFILTRATES IN PLASMODIUM FALCIPARUM PLACENTAL INFECTION

Jean-Marie Dangou; Annick D. M. Mansourou, Institut Pasteur, Dakar, Senegal; JeanYves Lehesran; Nadine Fievet, Institut de Recherche et Developpement, Dakar, Senegal; Ibrahima Gaye, Maternité Roi Baudoin, Guédiawaye, Dakar, Senegal

Background: The inflammatory process in placentas infected by Plasmodium falciparum is variable and a possible index of disease severity.

Design: 30 placentas with histopathologic lesions of malaria were assessed by immunohistochemistry using antibodies anti CD68, anti CD45 RO, anti CD20 cy, anti CD79á (DAKO SA), we identifying types of inflammatory cells, specifying their location and estimating their quantity.

Results: Placental infection was acute in $13 \%$ of cases, chronic but ongoing in $33 \%$ and remote in $54 \%$. In all placentas the majority of inflammatory cells were CD68+ (monocytes/ macrophages) associated with a secondary population of $\mathrm{T}$ lymphocytes $(\mathrm{CD} 45 \mathrm{RO}+)$ and a weak tertiary population of B lymphocytes (CD20cy+ or CD79á +$)$. The infiltrate made up of these cells localized in inter villous spaces and around syncytiotrophoblastic cells. Chronic malarial infection cases were associated with a massive inter villous infiltration by monocytes/macrophages and $\mathrm{T}$ lymphocytes. Remote infection was associated with a statistically significant decrease in the inflammatory infiltrate especially in quantities of monocytes/macrophages.

Conclusions: During malaria in pregnant women, the monocytes/macrophages and $\mathrm{T}$ lymphocyte component of inflammation is increased during active infections.

\section{CLINICAL AND PATHOLOGIC FINDINGS IN PLACENTAS WEIGHING MORE THAN 800 GRAMS \\ Peter Kragel, Brody School of Medicine, Greenville, NC, United States}

Background: Placentas weighing more than 800 grams have been associated with maternal and fetal pathology, including fetal hydrops and storage disorders, and maternal diabetes mellitus.

Design: Placentas weighing more than 800 grams were identified by an electronic search of surgical accession specimens received from 2003 to 2005 . The clinical and histopathologic findings were reviewed, and compared to a control group of randomly selected term placentas with weights less than 800 grams. Statistical significance was determined using the Chi Square Test.

Results: Thirty-two placentas weighing more than 800 grams were identified. The average maternal age at delivery was 27 years (range 16 years to 37 years). The average placental weight was 871 grams (range 801 grams to 996 grams). Associated conditions included maternal hypertension (19\%), diabetes mellitus (15\%), placental infarct $(22 \%)$ and chorioamnionitis $(28 \%)$. No evidence of a storage disorder or hydropic change was identified. The average maternal age at delivery in the control group was 26 years (range 13 years to 40 years). The average placental weight was 503 grams (range 286 grams to 760 grams). In the control group, associated conditions included maternal hypertension $(5 \%)$, diabetes mellitus $(2.5 \%)$, placental infarct $(7.5 \%)$, and chorioamnionitis $(25 \%)$. There were no statistically significant differences at the .05 level identified between the two groups. The $\mathrm{p}$ value was less than or equal to 0.10 for diabetes mellitus and maternal hypertension.

Conclusion: Placentas weighing more than 800 grams were associated with more maternal diabetes, maternal hypertension, and placental infarcts than the control group, but the differences were not found to be statistically significant. Maternal age was also not significantly different between the two groups. While placentas weighing more than 800 grams may be associated with certain maternal and fetal pathologic conditions, such as fetal hydrops, storage disorders, and maternal diabetes mellitus, and should receive careful histopathologic evaluation, such placentas are likely to reveal histopathologic features and have clinical associations similar to placentas of normal weight.

\section{PLACENTAL HISTOMORPHOLOGY IN INTRAUTERINE GROWTH RETARDATION}

Sonu Nigam, Maulana Azad Medical College, New Delhi, India; Kavita -; A K Mandal; Usha Manaktala, MAM College, N Delhi, India

Background: Intrauterine growth retardation (IUGR) is an important cause of fetal morbidity and mortality. A careful histopathological examination of placenta can explain the past events which determine the fetal out come as it is the major link between the mother and fetus.

Design: Thirty singleton placentas of IUGR pregnancies along with normal controls $(n=30)$ of $>28$ weeks gestation were included in this study. The gross and microscopic features of both groups were evaluated and compared.

Results: The average weight of IUGR placenta (230 gms, range: 200-230 gms) was significantly lower than control group (510gms, range: 480-540 gms). The number and extent of infarction was higher in IUGR group ( $>3$ in no., $>3 \mathrm{cms}$ size, in 19/30 IUGR $\mathrm{Vs}<3$ in no., $<2 \mathrm{cms}$, in 10/30 controls). On histology, chorioamnionitis was seen in $4 / 30$ IUGR and $0 / 30$ of the control group. Extensive intervillous deposition ( $>25 \%$ villi in $15 / 30$ cases vs. $<5 \%$ in $7 / 30$ controls), syncytial knotting ( $>30 \%$ villi in $20 / 30$ cases vs. $<30 \%$, $10 / 30$ controls), calcification $(>10 \%, 7 / 30$ cases vs. $<5 \%$ in $9 / 30$ cases $)$ and infarction
( $>25 \%$ villi in $15 / 30$ cases vs. $<15 \%$ villi in $8 / 30$ controls) were seen in IUGR group as compared to the control group. Stromal fibrosis was seen in $17 / 30$ IUGR and none of the control group.

Conclusion: All these histological changes were statistically significant. These changes indicate a reduction in blood flow to placenta leading to chronic placental insufficiency. This could be a major reason leading to IUGR. This information may be helpful in management of subsequent pregnancies and decreasing incidence of IUGR.

\section{STASIS-INDUCED FETAL THROMBOTIC VASCULOPATHY INCLUDES} CHORIONIC PLATE AND STEM VILLUS THROMBI

Wolfram Riedlinger, Children`s Hospital Boston, Boston, MA, United States; Mana Parast, Brigham and Women's Hospital, Boston, MA, United States; Theonia Boyd, Children's Hospital Boston, Boston, MA, United States

Background: We previously presented clinical and pathologic placental data from two independent series of late second and third trimester stillbirths attributable to umbilica cord compression ("umbilical cord accidents"). From those series, we now present data not initially reported that demonstrate chorionic plate and stem villi as the most sensitive sites for identifying thrombosis within the placental fetal vascular tree. We use this observation to expand the operational definition of fetal thrombotic vasculopathy.

Design: Placentas from stillbirths attributable to cord accidents from two institutions with placental and autopsy pathology were reviewed, from two different time periods. There were 49 cases from one institution, from 7/95-6/04. From the second institution there were 14 cases from 1/02-12/05, yielding 63 cases in total. The following histologic criteria were evaluated: location of fetal vascular thrombosis within muscularized umbilical cord, chorionic plate and/or stem villi; presence of terminal villus changes of "fetal thrombotic vasculopathy" (15 or more clustered avascular villi or villi with villousstromal karyorrhexis, in two or more independent locations); and gross placental findings predisposing to or indicating cord compression (flat cord, abnormal cord insertion, gross ectasia or thrombosis, hypercoiled or narrow cord). Clinical findings predisposing to cord compression (nuchal or body cord, oligohydramnios) were also tabulated.

Results: Chorionic plate and stem villus vessels were the most sensitive sites for detecting thrombi, present in $87 \%(55 / 63)$ and $83 \%(52 / 63)$ of cases, respectively. Umbilical cord thrombi were identified in less than half of cases $(43 \%, 27 / 63)$, and half as often as chorionic plate and stem villus thrombi. Fetal thrombotic vasculopathy was present in a regional distribution in $100 \%$ of cases (all 14) from one institution (noted frequently but not tabulated in the other series). Clinical and/or pathologic findings compatible with umbilical cord compression were present in 95\% (60/63) of cases.

Conclusion: Two independent institutions' experience with late second and third trimester stillbirth due to umbilical cord compression clearly identifies the chorionic plate and stem villus tributaries of the umbilical vessels as most commonly manifesting thrombosis, rather than the umbilical cord per se. We attribute this observation to two factors: the multitude of chorionic plate and stem villus vessels available for histologic evaluation; and more importantly, the angulated branching of these vessels, leading to physiologic blood flow turbulence which in turn renders them susceptible to localized thrombosis in the setting of upstream blood flow obstruction. The regional distribution of villous-stromal karyorrhexis and avascular villi within terminal villi also reflects patchy thrombosis of upstream chorionic and stem villi, and not only supports blood flow obstruction as the mechanism of demise but also distinguishes pathologic antenatal thrombosis from global passive involution of fetal placental vessels following demise. Finally, that thrombosis and terminal villous changes both reflect upstream blood flow obstruction makes it logical to broaden the definition of fetal thrombotic vasculopathy in the setting of non-acute umbilical blood flow obstruction, to encompass pathology within all levels of the placenta fetal vascular tree. We propose "stasis-induced fetal thrombotic vasculopathy" to describe this clinicopathologic condition.

\section{PLACENTAL MATURATION DEFECT: IMBALANCE OF GROWTH AND} ANGIOGENESIS IN CHORIONIC VILLI

Thomas Stallmach, Pathology, University Hopital Zürich, Zürich, Switzerland; Jörg Ersch, Neonatology, University Hospital Zürich, Zürich, Switzerland; Aleksandra Prtilo Faltermeier, Obstetrics, University Hospital Zürich, Zürich, Switzerland; Martina Storz, Pathology, University Hospital Zürich, Zürich, Switzerland; Ernst Beinder, Obstetrics, University Hospital Zürich, Zürich, Switzerland

Background: Maturation defect of the placenta kills 1.3 of 1000 babies in the last 4 weeks before birth due to hypoxia. There are typical morphological findings in terminal vill featuring a reduction in number and length of syncytiocapillary membranes. However many pathologists regard this reduced vascularity as post mortem artifact. Therefore, better criteria are needed to define the underlying arrest of angiogenesis.

Design: In a prospective study, ultrasonography was performed prior to planned caesarean section in order to correlate echogenicity of the placenta with histology of terminal villi after delivery. Placentae defined histologically as having maturation defect and controls were examined on one slide in the form of a tissue micro array. Tissue cores were retrieved from conventional slides in which the "most perfect areas" were selected. Four samples were taken from each placenta ( $2 \mathrm{x}$ subchorionic, $2 \mathrm{x}$ close to basal plate). Evaluation was done on hematoxylin-eosin stained slides by counting syncytiocapillary membranes per terminal villus and by different immunostains: p63 (as marker of cytotrophoblast nuclei), MIB1 (as marker for proliferating cytotrophoblast), inhibin (cytoplasmic staining in trophoblast, evaluated semiquantitatively as low versus high expression), beta-HCG (cytoplasmic in trophoblast, evaluated semiquantitatively as low versus high expression). Results: The mean number of syncytiocapillary membranes per terminal villus (counting the ten most developed villi in two samples from close to basal plate) was significantly lower $(\mathrm{p}<0.01)$ in maturation defect $(1.1$ versus 1.9$)$. A significant $(\mathrm{p}<0.05)$ higher number of cytotrophoblast nuclei was stained with MIB1 in maturation defect compared to control and significantly more placentae with maturation defect expressed inhibin "high". 
These differences were not present with comparison of subchorionic villi. Neither location showed differences for beta-HCG.

Conclusion: Placentae with maturation defect of terminal villi usually are of normal weight or even large. Nevertheless, they have a greater growth compartment as reflected by increased numbers of proliferating cytotrophoblast. This seems to be associated with a negative effect on development and differentiation of vessels as less of the circumference of each terminal villus is differentiated in the form of syncytiocapillary membranes. Placentae with maturation defect express more inhibin, which is adequate for placental tissue of younger gestational age. In conclusion, maturation defect shows (i) disturbed balance of cytotrophoblast growth and vascular differentiation in terminal villi, (ii) can be recognized by surrogate markers, and (iii) inhibin measured in maternal blood could eventually turn out as a marker of increasing risk of hypoxia to the fetus.

746 MICROSCOPIC CHORIONIC PSEUDOCYSTS IN PLACENTAL MEMBRANES: A HISTOLOGIC LESION OF IN-UTERO HYPOXIA

Jerzy Stanek, Children`s Hospital NHS Trust, Sheffield, United Kingdom; Eric Weng, University of Cincinnati College of Medicine, Cincinnati, OH, United States

Background: Subchorionic, septal and cell island cysts are often found in placentae that show increased perivillous fibrin, maternal floor infarction, or features of placental malperfusion. They are also more numerous in placentae from high altitudes. We have described histologically identical but grossly unapparent microscopic chorionic pseudocyst arising in the chorion laeve of placental membranes (mccpm). The current analysis aims to illustrate clinicopathologic correlations of mccpm on a large placental material.

Design: Placental and selected clinical parameters of all 172 consecutive placentae with mccpm (study group, sg) and all consecutive 3743 placentae without mccpm (control group, cg) from years 1994-2005 were extracted from our database and compared. Mean gestational age was 34.9 and 34.4 weeks in $\mathrm{sg}$ and $\mathrm{cg}$, respectively ( $>0.05$ ). Statistical analysis using Chi-square or ANOVA tests was performed where appropriate.

Results: By immunohistochemistry, mccpm are lined by fascin, placental alkaline phosphatase and E-cadherin-positive extravillous trophoblasts, and contain digested PAS, collagen IV and fibronectin-positive, and fibrin, laminin, fascin, E-cadherin, placental alkaline phosphatase, inhibin, and human chorionic gonadotropin-negative fibrinoid. Mccpm were observed in $4.3 \%$ of all placentae and in $14.9 \%$ of placentae from preeclamptic mothers from 24-42 weeks pregnancy, their gestational weeks ${ }_{i}{ }^{-}$distribution almost mirroring that of the distribution of preeclampsia, with a peak in the middle of third trimester. Mccpm were statistically significantly associated with preeclampsia $29.6 \mathrm{vs} 7.8 \%$ $(\mathrm{p}<=.001)$ and maternal diabetes mellitus 8.7 vs $5.1 \%(\mathrm{p}<=.05)$, sg and cg respectively. There were no statistically significant differences with chronic hypertension, maternal substance abuse, multiple gestations, and poor prenatal care. Statistically significant differences in placental histology in sg vs cg were found as follows, respectively (\%): decidual arteriolopathy 33.7 vs 14.6 ( $\mathrm{p}<=0.001$ ), heterogeneous maturation 13.9 vs 3.2 $(\mathrm{p}<=0.001)$, homogenous maturation 11.0 vs $2.9(\mathrm{p}<=0.001)$, global hypoxic pattern of placental injury 21.5 vs 11.7 ( $<<=0.001)$, infarction 22.7 vs $14.2(p<=0.01)$, chorangiosis 18.0 vs 7.8 ( $p<=0.001$ ), laminar necrosis of membranes 22.1 vs 9.0 ( $p<=0.001)$, obliterative endarteritis of stem villi 5.8 vs 1.7 ( $p<=0.001$ ), erythroblastosis of fetal blood 4.1 vs 1.7 ( $\mathrm{p}<=0.05)$, decidual hemosiderosis 2.9 vs $1.1(\mathrm{p}<=0.05)$, acute chorioamnionitis 13.9 vs 35.6 ( $\mathrm{p}<=0.001$ ), vilous fibrosis 2.9 vs 7.3 ( $\mathrm{p}<=0.05$ ), and villous oedema 1.6 vs $4.4 \%$ $(\mathrm{p}<=0.05)$. There were no statistically significant differences $(\mathrm{p}>0.05)$ with meconium staining, retroplacental hematoma, perivillous fibrin deposition, intervillous thrombi, chronic villitis, chorangiomas, placenta accreta, amnion nodosum, and marginate/vallate placenta.

Conclusion: Highly statistically significant associations of mccpm with preeclampsia and a cluster of placental lesions known to be linked to placental insufficiency (abnormal maturation, decidual arteriolopathy, global villous hypoxic pattern, chorangiosis, infarction, membrane laminar necrosis, villous obliterative endarteritis) suggest that the formation of mccpm is a result of degenerating processes due to malnutrition and hypoxia in the migrating extravillous trophoblasts of the chorion leave, particularly in preeclampsia. Mccpm should therefore be regarded and reported as a hypoxia-associated placental lesion.

\section{Proteomics}

747 PROTEOMIC ANALYSIS OF METASTASIS-ASSOCIATED PROTEINS OF HUMAN COLORECTAL CARCINOMA CELL LINES WITH DIFFERENTIAL METASTATIC POTENTIALS

Zhao Liang; Ding Yanqing, Southern Medical University, Guangzhou, Guangdong, China Background: Metastasis is a common phenomenon and the major lethal cause of cancers. So far, however, little has been known about how tumorigenic cells acquire the ability to become invasive to initiate metastasis because tumor metastasis is a complex malignant process. Most studies on metastasis have usually focused on the genetic changes of related molecules or a few proteins, which made the metastatic mechanism remain poorly elucidated. Among the currently available techniques, proteomics ones allow for identification of the protein changes caused by the disease process in a relatively highthroughput manner.

Design: To better comprehend the mechanism underlying colorectal carcinoma (CRC) metastasis and to search for potential markers for predicting CRC metastasis, two CRC cell lines with different metastatic potentials were investigated by phenotypic analyses and proteomics technologies. First, a pair of highly and poorly metastatic subline(termed SW620 and SW480, respectively), originating from the same patient, was identified by spontaneous tumorigenicity and metastasis in vivo and characterized by metastatic phenotypes analysis in vitro. The surgical orthotopic implantation (SOI) technique was originally used to develop a reproducible colorectal cancer model in nude mice with stable tumor growth and metastasizing course. Subsequently, a proteomic approach, using two- dimensional gel electrophoresis followed by matrix-assisted laser desorption/ time of flight mass spectrometry, was used to compare the protein expression profiles between SW620 and SW480 cell lines. The differential proteins were identified and further verified by Western blotting, immunohistochemistry and semiquantitative reverse transciptase polymerase chain reaction analysis.

Results: Phenotypic analyses in vivo and in vitro showed that the SW480 cells from the primary tumor showed more highly metastatic potential to distant organs than SW620 cells from a lymph node metastasis which possess more highly lymph node metastatic potential. Our findings indicate that two cell lines of the same genetic background but with different metastatic potential are still valuable models for investigation on colorectal cancer metastasis. Furthermore, ten proteins displaying significant and reproducible differences between the two metastatic cell lines were successfully identified by comparative proteomic analysis and were further verified at the protein and mRNA levels. Of the identified proteins, the expressions of phosphoglycerate mutase, phosphatidylethanolamine binding protein and high-mobility group box 1 were elevated in SW620 cells. However, heat shock protein 27 , annexin I, methylthioadenosine phosphorylase, cofilin-1 and epidermal fatty acid binding protein were down-regulated in SW620 cells. Our immunohistochemistry assays of colorectal cancer revealed that HSP27 overexpression was related to lymphatic metastasis

Conclusion: The present study made a systematic research on tumor metastasis by phenotypic analyses and comparative proteome technique using SW480 and SW620 as metastatic model. Ten metastasis-associated proteins were identified and most of these identified proteins have been reported to be associated with tumor metastasis. HSP27 may be a key factor for preventing tumor metastasis and a therapeutic target for the treatment of CRC patients with metastasis.

\section{PLASMA MEMBRANE PROTEIN IDENTIFICATION BY CELL SURFACE BIOTINYLATION AND TANDEM MASS SPECTROMETRY OF CLINICAL SPECIMENS}

Wilfrido Mojica; Jianlan Sun, University at Buffalo, Buffalo, NY, United States

Background: Although plasma membrane proteins account for only $30 \%$ of a cell's overall protein composition, they represent more than two thirds of the current protein targets for today's drugs. Current analysis of plasma membrane proteins is hindered by their low abundance and insoluble nature. Identification of differentially expressed plasma membrane proteins between normal and neoplastic cell populations would substantially contribute to our knowledge of cell biology, as well as enhance and possibly accelerate our ability to diagnose, classify and potentially treat diseased cells. Identification of plasma membrane proteins directly from clinical samples would hasten the development of translational medicine.

Design: Colonic epithelial cells were procured from an adenocarcinoma containing hemicolectomy specimen excised for curative purposes. Separately, normal colonic epithelial cells and neoplastic colonic epithelial cells were obtained by manual exfoliation and enriched for by immunomagnetic bead separation (Dynal Epithelial Enrich, Invitrogen, Deer Park, WI). Surface plasma membrane proteins from normal and neoplastic populations were isolated by selective biotinylation and strepavidin mediated purification using the PinPoint Cell Surface Isolation Kit (Pierce, Rockford, IL). Recovered plasma membrane proteins were resolved by sodium dodecyl sulfate polyacrylamide gel electrophoresis (SDSPAGE) and visualized by silver staining. Differentially expressed protein bands were excised, reduced, alkylated and digested overnight with trypsin. Recovered proteolytic fragments were analyzed by liquid chromatography tandem mass spectrometry (Custom Biologics, Toronto, ON). Protein identifications were made by cross correlating $\mathrm{ms} / \mathrm{ms}$ spectra against a non-redundant database downloaded from the National Center for Biotechnology Information (NCBI) using the SEQUEST Algorithm (Termo, CA).

Results: Sufficient cells were procured from the clinical specimen for surface protein biotinylation. Proteins eluted from the surface membrane and analyzed by SDSPAGE demonstrated four distinct bands present in the neoplastic colonic cells not present in the normal colonic cell lane. Peptide mass sequencing by LC/MS/MS identified the presence of desmoglein-1 in the neoplastic group. A Medline literature search revealed no previously reported association between desmoglein-1 and colon cancer.

Conclusion: Using a protocol for positive cell enrichment, plasma membrane surface biotinylation and mass spectrometry, exploration of the plasma membrane proteome from clinical samples is feasible. This approach may yield the identification of additional previously undocumented proteins from human neoplastic cells whose ultimate value may be in diagnosis, prognosis or therapy.

749 DIFFERENTIAL PHOSPHOPROTEIN DETECTION OF CLINICAL TISSUE USING TWO DIMENSIONAL LIQUID CHROMATOGRAPHY TANDEM MASS SPECTROMETRY

Wilfrido Mojica; Elizabeth Marchetti, University at Buffalo, Buffalo, NY, United States Background: Protein phosphorylation plays an integral regulatory role in cellular signaling and pathogenesis. The ability to perform large scale identification of phosphorylated proteins from clinical samples could yield significant findings in our understanding of human disease. However, the identification of phosphorylated proteins is currently hindered by both their overall low abundance in cells and low throughput technology. In addition, phosphorylated proteins in clinical samples are subjected to extensive degradation when exposed to fixatives. The feasibility of overcoming these obstacles was examined utilizing manual exfoliation with immunomagnetic bead separation to obtain pure cell populations from fresh clinical specimens, followed by whole cell lysis, phosphopeptide enrichment and detection using two dimensional liquid chromatography, tandem mass spectrometry (2D-LC/MS/MS).

Design: A hemicolectomy specimen containing adenocarcinoma of the colon and excised for curative purposes was obtained at the time of extirpation. Separately, normal and neoplastic cells were exfoliated from the tissue and subjected to positive enrichment 